\title{
Tunning Hydrogen Storage Properties of Carbon Ene-Yne (CEY) Nanosheets through Selected Foreign Metal Functionalization
}

Ekaterina Anikina ${ }^{1,2, *}$, Tanveer Hussain ${ }^{3, *}$, Valery Beskachko ${ }^{1}$, Hyeonhu Bae ${ }^{4}$, Hoonkyung Lee ${ }^{4}$, Rajeev Ahuja 2,5

Institute of Natural Sciences and Mathematics, South Ural State University, 76, Lenin Prospekt, Chelyabinsk 454014, Russia

${ }^{2}$ Materials Theory Division, Department of Physics and Astronomy, Uppsala University, Box 516, 75120

Uppsala, Sweden

${ }^{3}$ School of Molecular Sciences, The University of Western Australia, Perth, WA 6009, Australia

${ }^{4}$ Department of Physics, Konkuk University, Seoul 05029, Republic of Korea

${ }^{5}$ Applied Materials Physics, Department of Materials and Engineering, KTH Royal Institute of Technology, S-100 44 Stockholm, Sweden

*Corresponding authors: anikate@,inbox.ru, tanveer.hussain@physics.uu.se 
Table S1. Basis set parameters and delta test (SIESTA/VASP)

\begin{tabular}{|c|c|c|c|c|c|c|}
\hline Orbital & $\mathrm{C}(2 \mathrm{~s})$ & $\mathrm{C}(2 \mathrm{p})$ & $\mathrm{H}(1 \mathrm{~s})$ & $\mathrm{Na}(2 \mathrm{~s})$ & $\mathrm{K}(3 \mathrm{~s})$ & $\mathrm{Ca}(3 \mathrm{~s})$ \\
\hline$r$, Bohr & 8.66 & 7.64 & 6.05 & 12.21 & 9.08 & 12.54 \\
\hline$r_{\mathrm{m}}$, Bohr & 2.81 & 3.27 & 2.52 & 8.39 & 8.85 & 6.88 \\
\hline$\Delta$, meV/atom & \multicolumn{2}{|c|}{$0.37 *$ (for bulk reference) } & --- & 0.69 & 0.73 & 34.0 \\
\hline relative $\Delta, \%$ & \multicolumn{2}{|l}{$2.9 *$ (for bulk reference) } & --- & 45.4 & 48.5 & 105.3 \\
\hline
\end{tabular}

a)

top view:

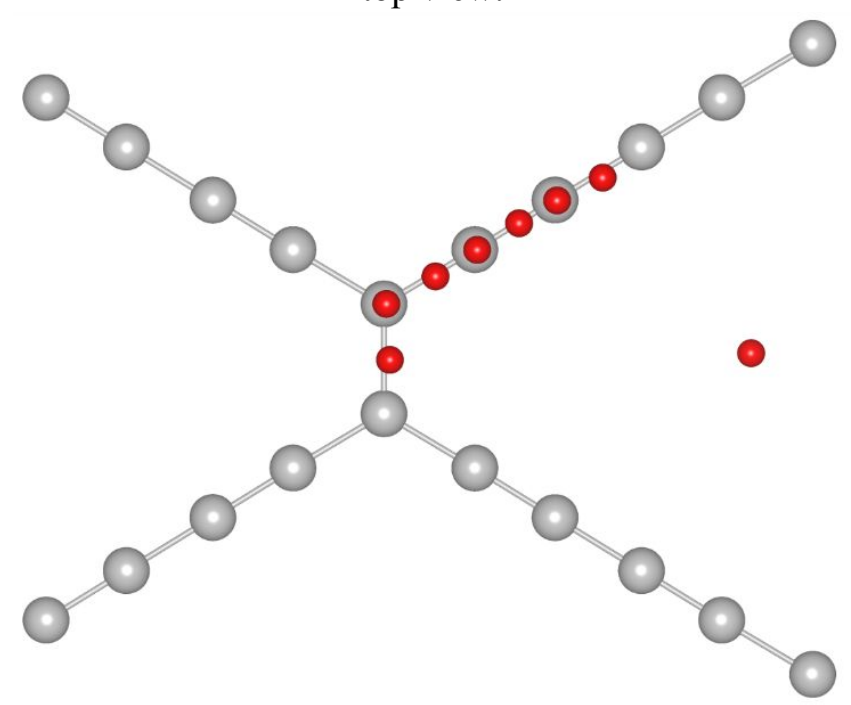

side view:

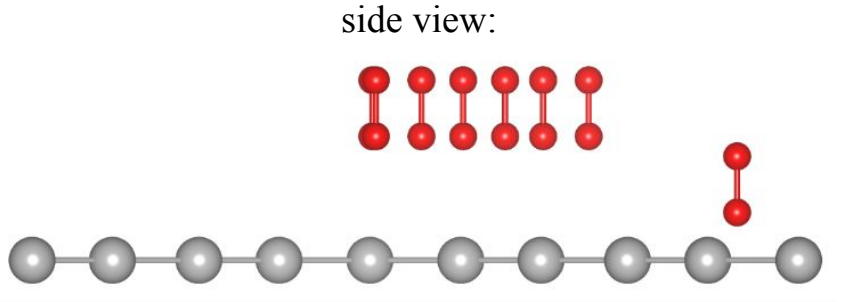

b)

top view:

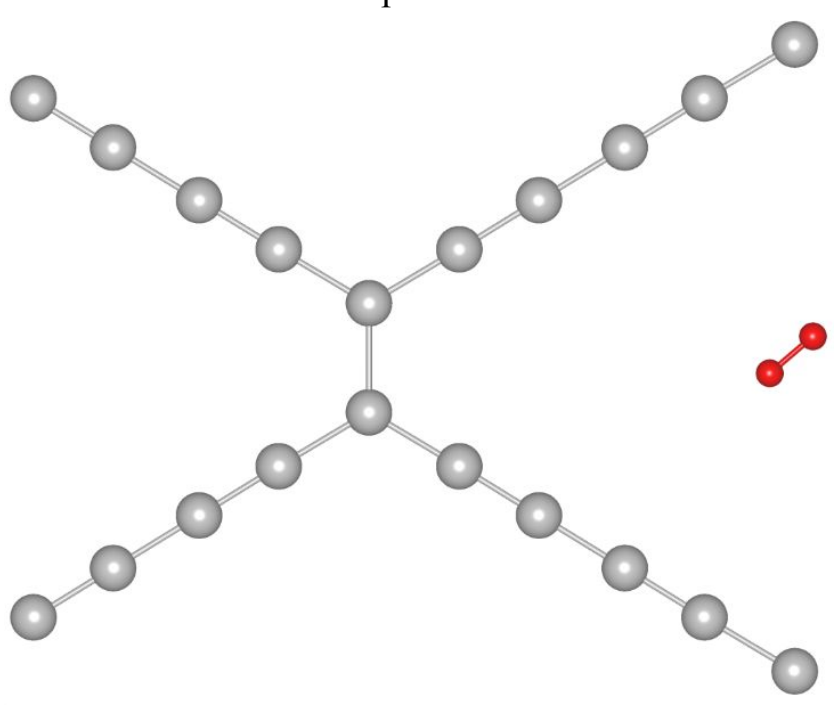

side view:

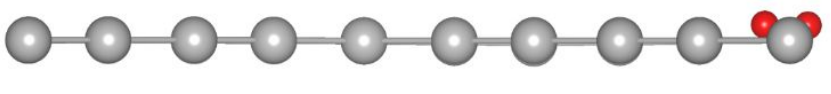

Figure S1. The geometry of hydrogenated pristine CEY: $a$ ) starting configurations of a single hydrogen molecule (only perpendicular to the CEY sheet positions are presented; parallel configurations were also considered); $b$ ) the most energetically favorable configuration obtained after the geometry relaxation. Carbon and hydrogen atoms are gray and red, respectively 
Table S2. Hydrogen adsorption energies (meV) on pristine CEY

\begin{tabular}{|c|c|c|c|c|c|c|}
\hline Package & \multicolumn{4}{|c|}{ SIESTA } & \multicolumn{2}{c|}{ VASP } \\
\hline XC & GGA & vdW-BH & DFT-D2 & LDA & DFT-D2 & DFT-D3 \\
\hline$E_{\text {bind }}$ & 12 & 48 & 64 & 104 & 68 & 88 \\
\hline
\end{tabular}

top view:

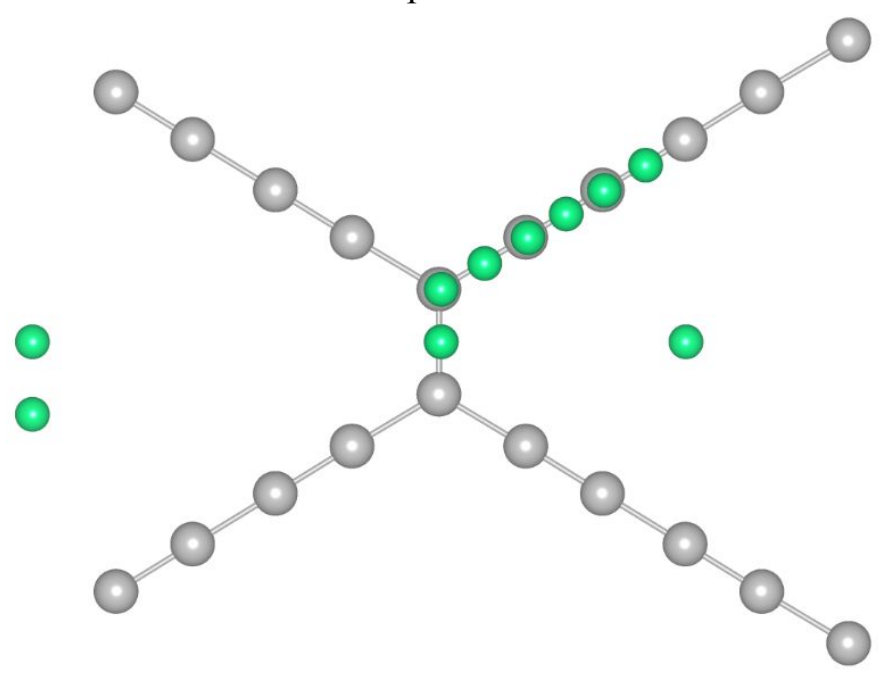

side view:

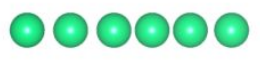

$\odot$

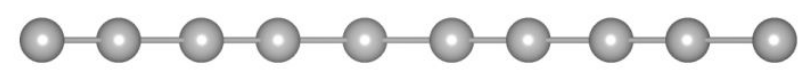

Figure S2. Starting positions of metal adatoms on CEY. Carbon atoms are gray, metal adatoms are green

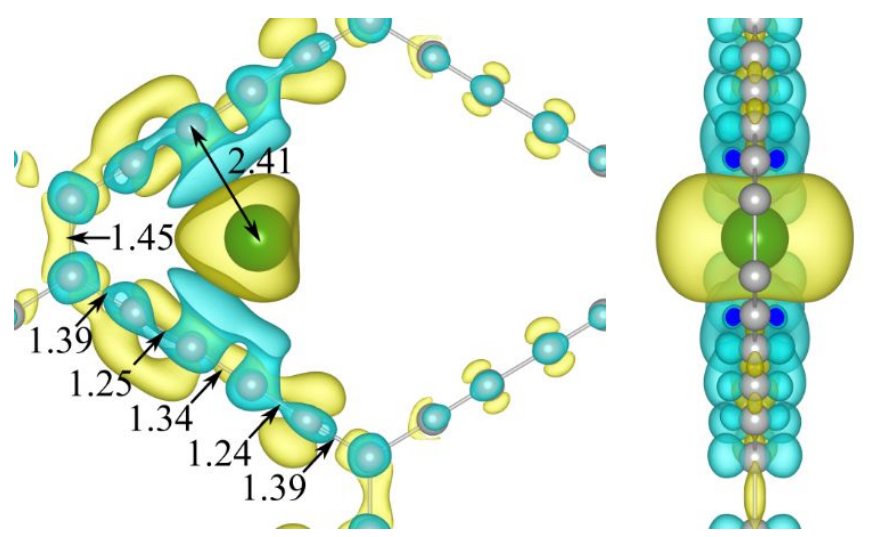

Figure S3. Optimized structure (VASP, DFT-D3) of CEY decorated with $\mathrm{Mg}$. C and $\mathrm{Mg}$ atoms are gray and green, respectively. All bond lengths and distances are in $\AA$. Yellow and cyan isosurfaces $(0.0015 e)$ show charge depletion and accumulation, respectively 
Table S3. Binding energy of metal atoms on CEY

\begin{tabular}{|c|c|c|c|}
\hline \multirow{2}{*}{ Me atom } & \multicolumn{2}{|c|}{ SIESTA } & VASP \\
\cline { 2 - 4 } & GGA & LDA & DFT-D3 \\
\cline { 2 - 4 } & $E_{\text {bind }}, \mathrm{eV}$ & $E_{\text {bind }}, \mathrm{eV}$ & $E_{\text {bind }}, \mathrm{eV}$ \\
\hline $\mathrm{Na}$ & 2.06 & 2.42 & 2.10 \\
\hline $\mathrm{K}$ & 2.81 & 3.15 & 2.67 \\
\hline $\mathrm{Ca}$ & 1.16 & 1.64 & 2.64 \\
\hline
\end{tabular}

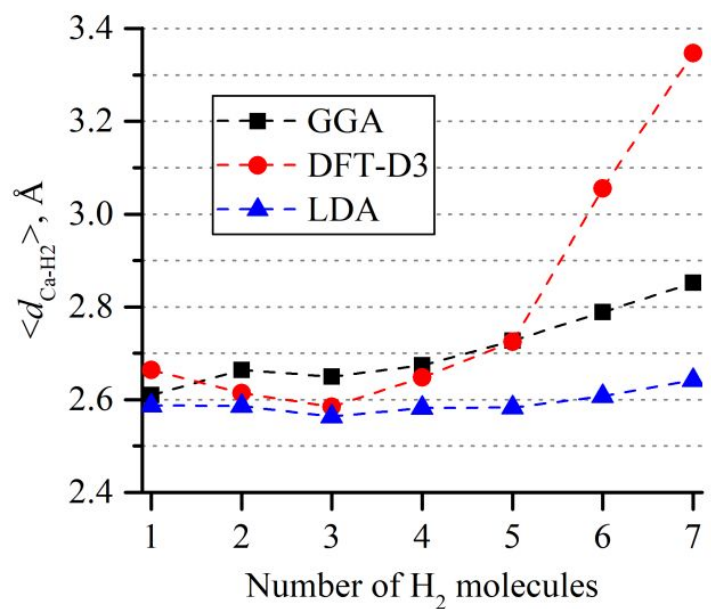

a)

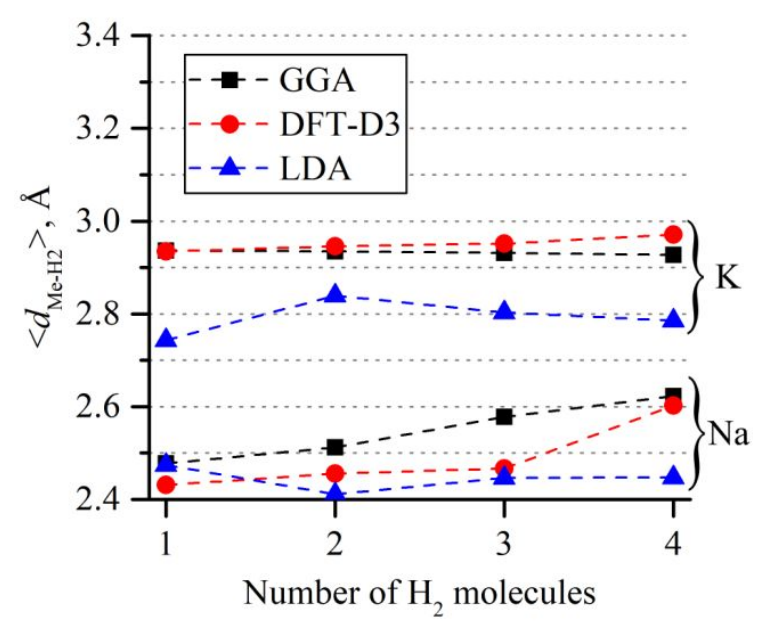

b)

Figure S4. The average distance between the metal adatom and the middle of a hydrogen molecule for $a$ ) CEY@Ca, b) CEY@Na and CEY@K. Blue triangles note the LDA (SIESTA) results, red circles - DFT-D3 (VASP) results, and black squares - GGA (SIESTA) results

Comments to Fig.S4. For $k \geq 6$ SIESTA and VASP calculations gave different relaxed geometries for hydrogenated CEY@Ca. In PAW simulations the sixth and seventh $\mathrm{H}_{2}$ molecule moved away from Ca (Fig. 3f,g), while in the SIESTA calculations all seven $\mathrm{H}_{2}$ molecules were located close to $\mathrm{Ca}$ upon geometry relaxation, therefore, we did not get a surge in $\left\langle d_{\mathrm{Ca}-\mathrm{H}_{2}}\right\rangle$ for $k=6$ and 7 as in DFT-D3 calculations. For CEY@Na and CEY@K, both packages yielded similar geometries of $\mathrm{H}_{2}$ molecules (Fig. S4b). Overall, Fig. S4 shows that LDA gave the lowest $\mathrm{H}_{2}$-Me distances (in all considered cases except CEY@Na $+1 \mathrm{H}_{2}$, where DFT-D3 simulation gave the lowest $\left\langle d_{\mathrm{Na}-\mathrm{H}_{2}}\right\rangle$ ). And for all metals and $k \leq 4$ we see a good agreement between the SIESTA and VASP geometries.

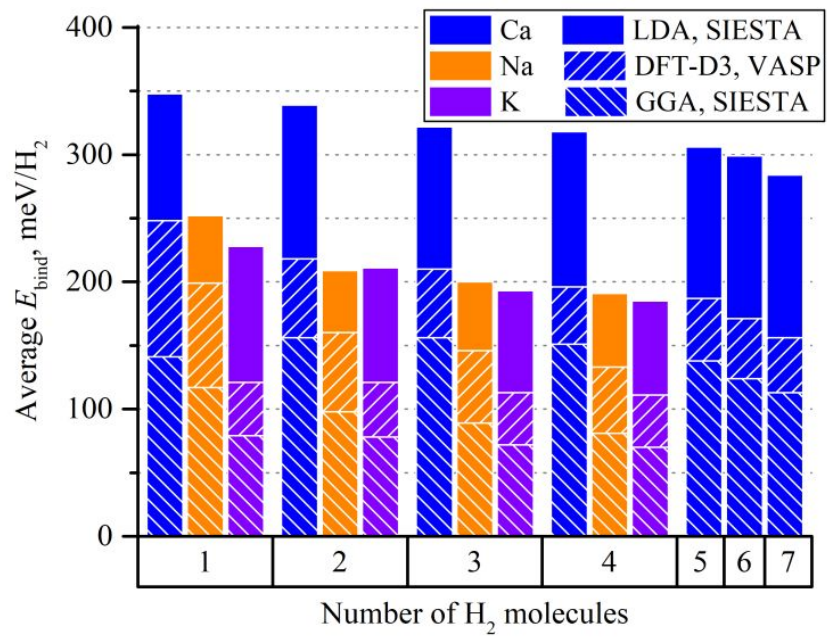

Figure S5. Dependence of average hydrogen binding energy on number of $\mathrm{H}_{2}$ molecules, $k$, for decorated CEY (concentration $\mathrm{C}_{20} \mathrm{Me}$ ): blue, orange, and purple columns present results for CEY@Ca, CEY@Na, and CEY@K, 
respectively
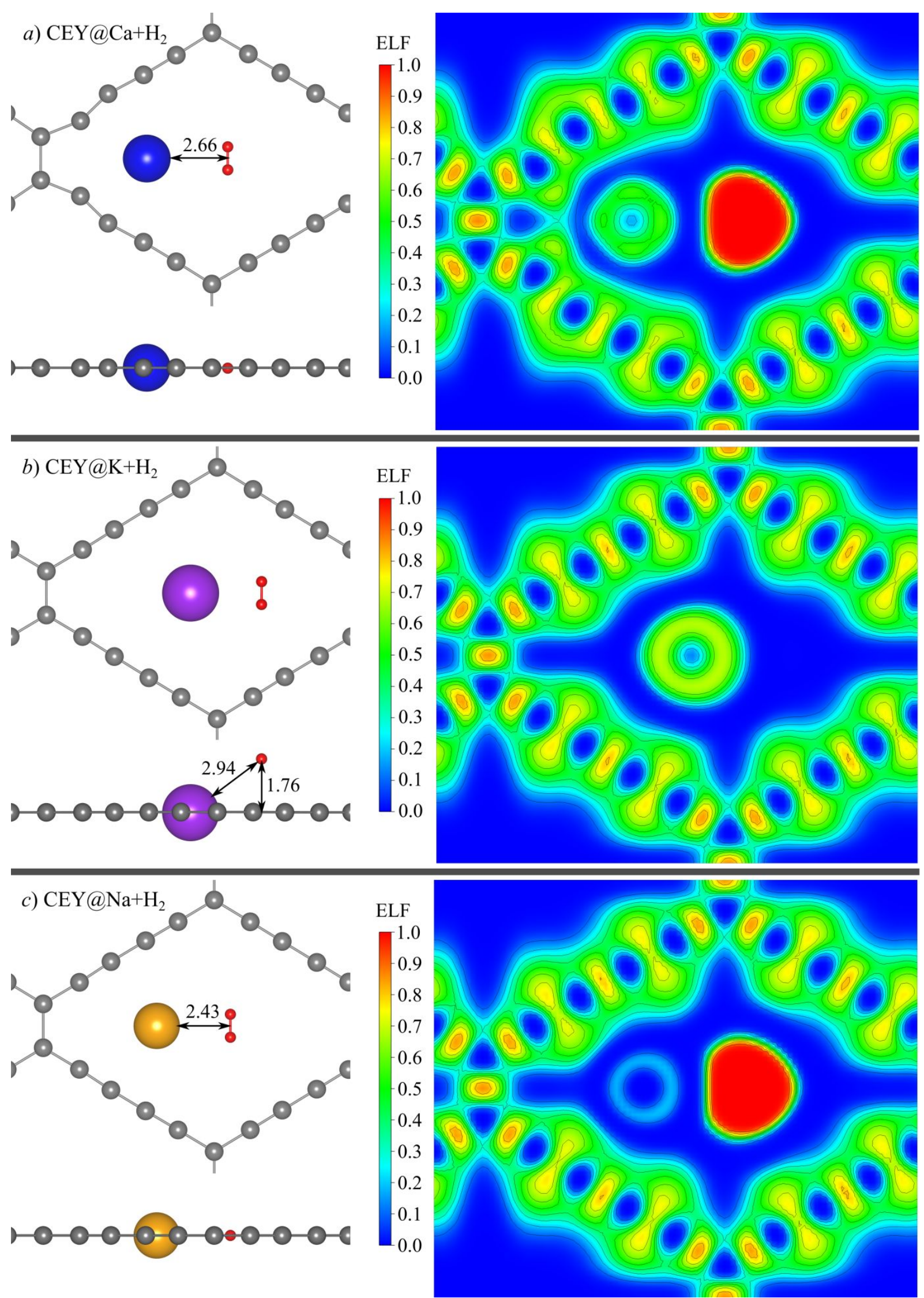

Figure S6. Electron localization function of hydrogenated CEY, decorated with $a$ ) $\mathrm{Ca}, b) \mathrm{K}$, and $c$ ) $\mathrm{Na}$. Calculated ELF maps are drawn for slices, perpendicular to the $z$-axis and containing Me atoms. Gray, blue, purple, and orange spheres represent $\mathrm{C}, \mathrm{Ca}, \mathrm{K}$, and $\mathrm{Na}$ atoms, respectively. All distances are in $\AA$ 
a)

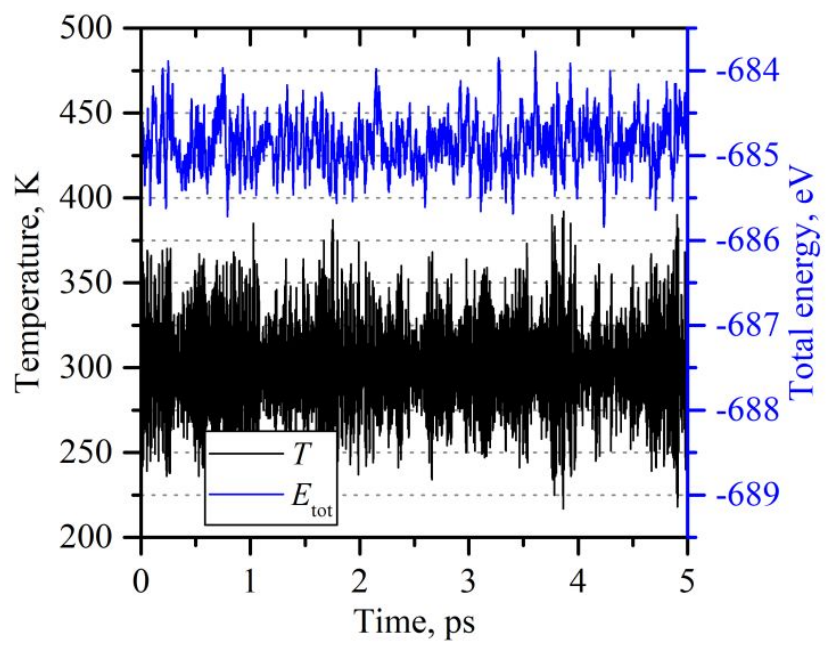

c)

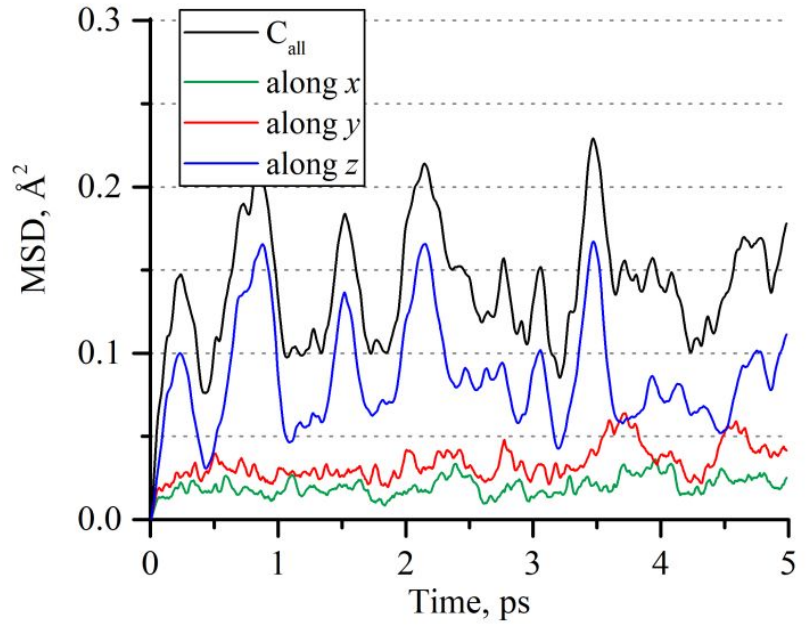

b)

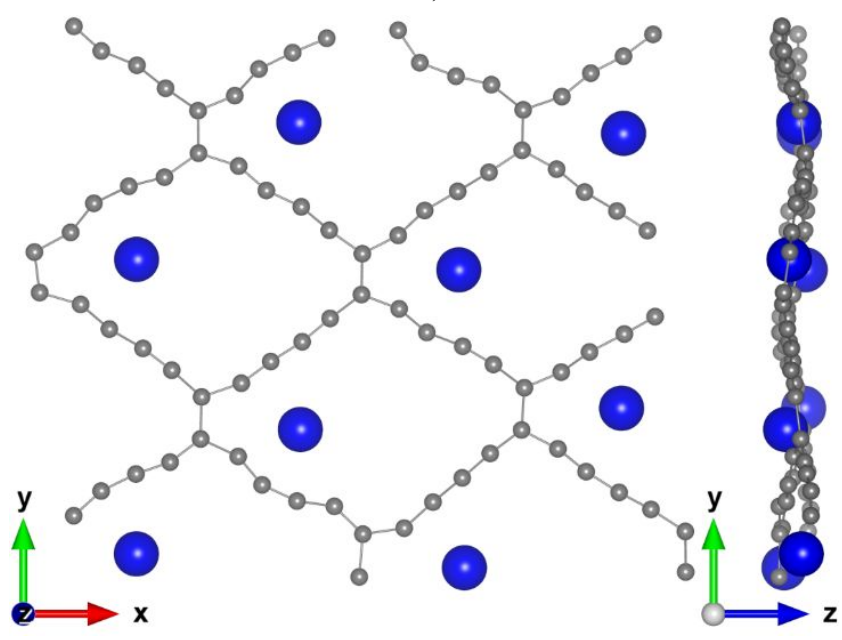

d)

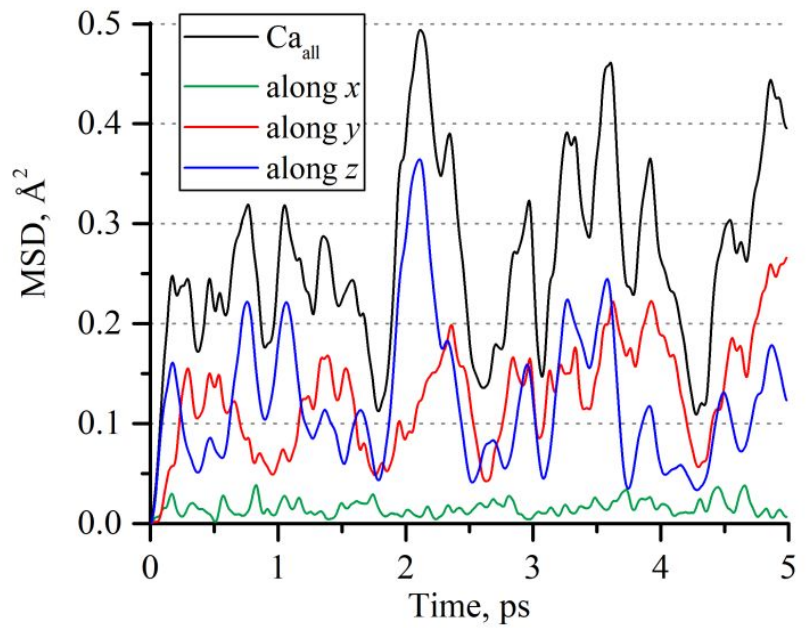

Figure S7. Production AIMD run at $300 \mathrm{~K}$ (NVT ensemble) of CEY@Ca $\left(\mathrm{C}_{10} \mathrm{Ca}\right.$ concentration): $\left.a\right)$ dependence of CEY@Ca temperature and total energy on the simulation time; $b$ ) snapshot of the structure (top and side views) after $5 \mathrm{ps}$ ( $\mathrm{C}$ and $\mathrm{Ca}$ atoms are gray and blue, respectively); element-wise decomposed mean square displacement for $c$ ) carbon and $d$ ) calcium

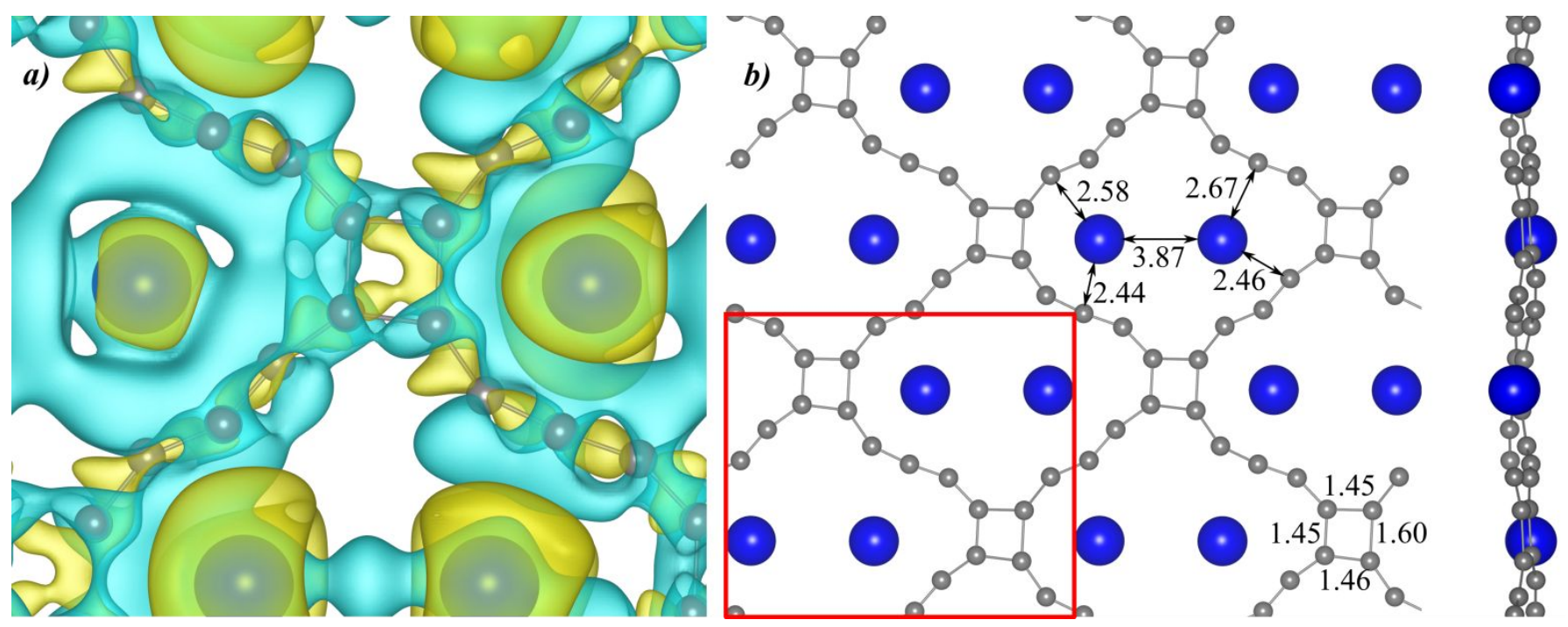

Figure S8. Optimized structure (VASP, DFT-D3) of Ca-decorated CEY $\left(\mathrm{C}_{5} \mathrm{Ca}\right): a$ ) charge distribution, and $b$ ) geometrical parameters. $\mathrm{C}$ and $\mathrm{Ca}$ atoms are gray and blue, respectively. The boundary of a computational cell is marked by the solid red line. All bond lengths and distances are in $\AA$. Yellow and cyan isosurfaces $(0.0015 e)$ show 
charge depletion and accumulation, respectively

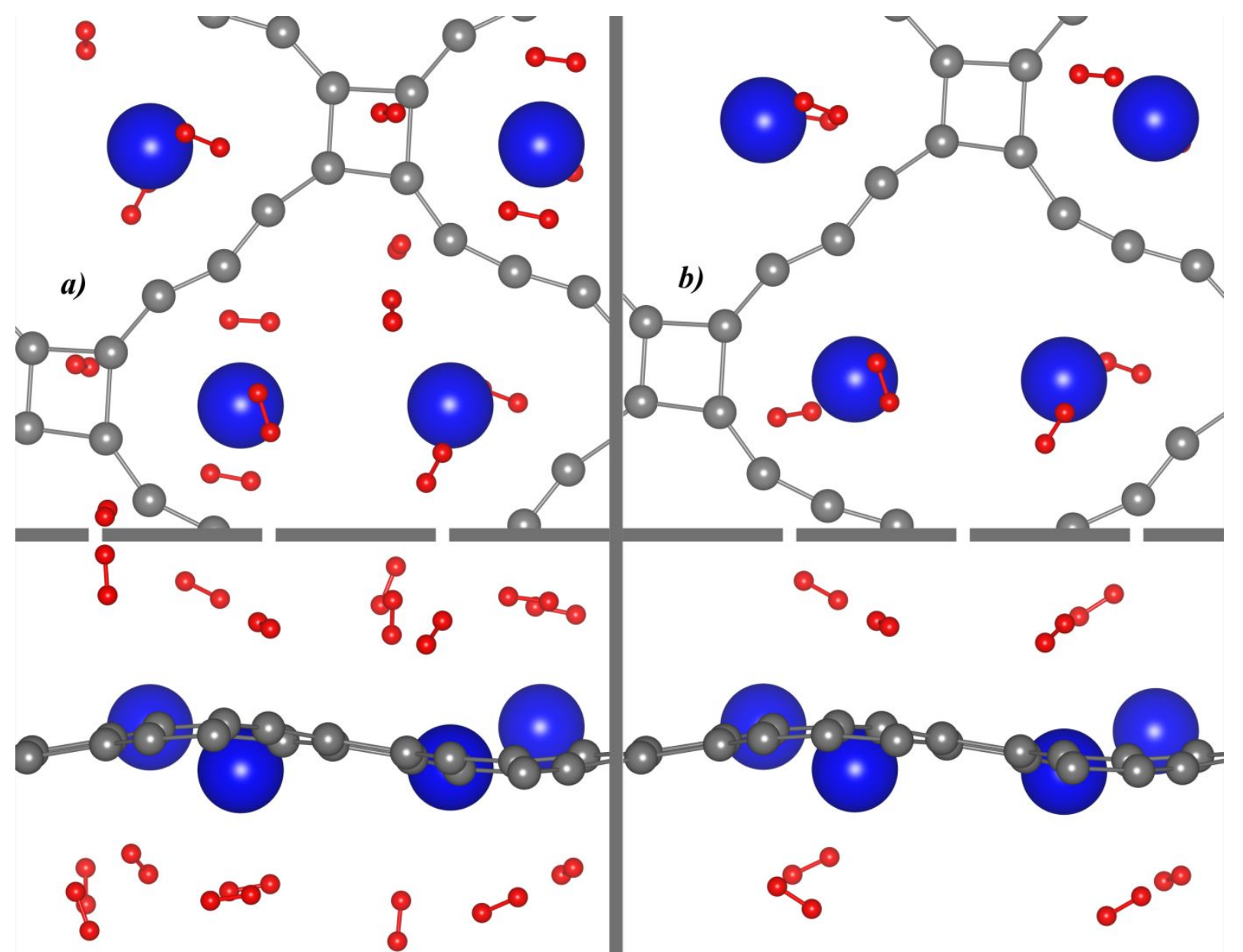

Figure S9. Relaxed structures (VASP, DFT-D3) of hydrogenated Ca-decorated CEY $\left.\left(\mathrm{C}_{5} \mathrm{Ca}\right): a\right) \mathrm{C}_{5} \mathrm{Ca}+4 \mathrm{H}_{2}\left(16 \mathrm{H}_{2}\right.$ molecules in the computational cell), and $b) \mathrm{C}_{5} \mathrm{Ca}+2 \mathrm{H}_{2}\left(8 \mathrm{H}_{2}\right.$ molecules in the computational cell). $\mathrm{C}, \mathrm{Ca}$, and $\mathrm{H}$ atoms are gray, blue, and red, respectively

Table S4. Parameters of hydrogen adsorption in the structure $\mathrm{C}_{5} \mathrm{Ca}+k \mathrm{H}_{2}$

\begin{tabular}{|c|c|c|c|c|}
\hline$k$ & av $E_{\text {bind }}, \mathrm{meV} / \mathrm{H}_{2}$ & $\mathrm{ad} E_{\text {bind }}, \mathrm{meV} / \mathrm{H}_{2}$ & average $T_{D}, \mathrm{~K}($ at $p=1 \mathrm{~atm})$ & $\mathrm{H}_{2}$ uptake, $\mathrm{wt} \%$ \\
\hline 4 & 126 & 105 & 106 & 7.4 \\
\hline 3 & 133 & 118 & 112 & 5.7 \\
\hline 2 & 141 & 129 & 118 & 3.8 \\
\hline 1 & 153 & 153 & 128 & 2.0 \\
\hline
\end{tabular}

